\title{
MS/MS of Ions in a Low Pressure Linear Ion Trap using a Pulsed Gas
}

\author{
Bruce A. Collings and Matthew A. Romaschin \\ MDS Analytical Technologies, Concord, Ontario, Canada
}

A pulsed valve was used to increase the pressure within the trapping region of a low-pressure linear ion trap by situating the pulsed valve close to the ion trapping region. The pressure was estimated to increase from a background pressure of $3.5 \mathrm{e}-5$ Torr of nitrogen to $0.49 \mathrm{mTorr}$ at the center of the trap. The increased pressure allowed excitation periods to be reduced from 100 to 25 ms without suffering losses in MS/MS efficiency during dipolar excitation. The reduction in excitation period translates into an increase in the overall duty cycle of the scan. (J Am Soc Mass Spectrom 2009, 20, 1714-1717) (C) 2009 American Society for Mass Spectrometry

$\mathrm{T}$ he in-trap fragmentation of ions in a low-pressure $(<5 \mathrm{e}-5$ Torr) linear ion trap (LIT), such as the 4000 QTRAP hybrid linear ion trap triple quadrupole mass spectrometer, require excitation periods typically ranging from 50 to $200 \mathrm{~ms}$. This is due to the low collision rates between the ion and the background nitrogen which are typically about a few collisions every millisecond [1]. Low-pressure LITs rely on the presence of higher order fields to keep ions confined during the excitation process and, as a result, it is necessary to use excitation amplitudes ranging from 10 to $100 \mathrm{mV}$ to reduce the degree of ion ejection. Increasing the higher order field content allows the use of higher excitation amplitudes [2,3]. The degree of ejection from an LIT can also be reduced by increasing the drive frequency of the LIT [1]. This leads to an increase in the pseudo-potential well depth, which allows for improved radial confinement. It is also known that the degree of ion ejection during the excitation process can further be reduced by operating at higher pressures [4].

In the case of the hybrid linear ion trap triple quadrupole mass spectrometer, the final mass analyzing quadrupole also serves as the LIT. In this type of instrument it is necessary that the pressure in the quadrupole be appropriate for both operation as a mass analyzing quadrupole ( $<5 \mathrm{e}-5$ Torr) and as a LIT.

The overall MS/MS efficiency at short excitation times can be increased by introducing a pressure increase during the excitation process with the use of a pulsed valve. The higher gas density results in an increased collision rate, leading to a more rapid conversion of kinetic energy to internal energy of the ion of interest. Using pulsed valves to vary the pressure and type of gas during the excitation process has been

Address reprint requests to Dr. B. A. Collings, MDS Analytical Technologies, 71 Four Valley Dr., Concord, Ontario L4K 4V8, Canada. E-mail: Bruce.Collings@sciex.com implemented and studied by a number of groups for both 3D quadrupole ion traps and Fourier transform mass spectrometers $[5,6]$. In each of the above cases conductance from the valve aperture to the ion trap was a limiting factor.

In the work presented here, experiments using a pulsed valve to increase the pressure locally within a low-pressure LIT are presented and discussed. The aperture of the pulsed valve is located next to the electrodes of the LIT and the temporal pressure profiles are measured using a fast ionization gauge. MS/MS efficiencies for the $m / z 138 \rightarrow 110$ transition of caffeine as a function of excitation amplitude, excitation period, and pressure are examined.

\section{Experimental}

Experiments were carried out on a hybrid linear ion trap/triple quadrupole mass spectrometer research breadboard that was a precursor to the QTRAP 5500 System (Applied Biosystems/MDS Analytical Technologies, Concord, ON, Canada).

A set of auxiliary electrodes were installed around the Q3 quadrupole rods [7]. The electrodes were 175 $\mathrm{mm}$ long and extended from the entrance of the quadrupole to $28 \mathrm{~mm}$ from the exit end of the quadrupole. A dc potential of $+1000 \mathrm{~V}$ was applied to the auxiliary electrodes causing the ions to be moved and confined axially within the last $28 \mathrm{~mm}$ of the Q3 quadrupole between the auxiliary electrodes and a gridded exit lens, Figure 1 . The ions were confined radially by the rf fields of the LIT. The LIT operated at a drive frequency of $1.228 \mathrm{MHz}$.

Ions of interest were injected into the LIT at Mathieu $q=0.6$. The ions were cooled for $20 \mathrm{~ms}$ before isolation using a $1 \mathrm{~ms}$ application of resolving DC applied at $q=$ 0.704 with the resolving DC adjusted to give an isolation window of $\sim 4 \mathrm{Da}$. The isolation window served to 
Q3/LIT Side View

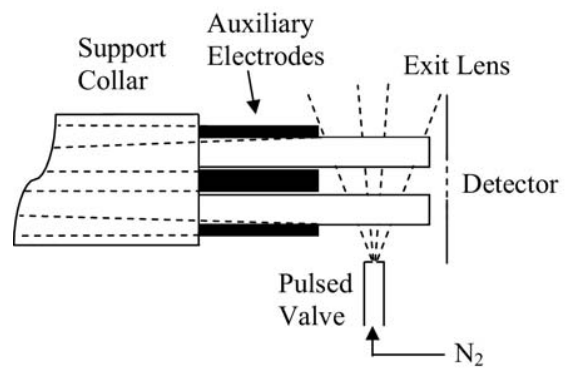

Figure 1. A detailed schematic of the trapping region in the Q3 quadrupole showing the relative locations of the pulsed valve and the auxiliary electrodes.

remove any unwanted ions in the LIT. The ions were then allowed to cool a further $50 \mathrm{~ms}$ before dipolar excitation. The extended cooling period was necessary to ensure that the ion of interest was fully cooled before excitation. After excitation the ions were allowed to cool for a period of $100 \mathrm{~ms}$ before scanning the ions out of LIT. In commercial practice the $100 \mathrm{~ms}$ cooling period is not required and is replaced by a shorter cooling period. Ions were scanned out of the LIT at a rate of $1000 \mathrm{Da} / \mathrm{s}$ using the technique of mass selective axial ejection (MSAE) [8]. In trap mode the pressure in the LIT was maintained at a base pressure of 3.5e-5 Torr of nitrogen.

The experimental set-up included a pulsed valve (The Lee Co., Westbrook, CT, USA, valve model INKA2437210H controlled with a spike and hold driver model IECX0501350A) that was backed with 90 Torr of nitrogen. The backing pressure was chosen such that the combination of opening the pulsed valve for long excitation periods with short scan times did not cause the overall average pressure in the chamber to rise above $9.5 \mathrm{e}-5$ Torr. The valve outlet aperture was 0.076 $\mathrm{mm}$ in diameter and was located $11 \mathrm{~mm}$ from side of the quadrupole rods and was directed towards the center of the LIT, Figure 2. The aperture of the pulsed valve was located $22 \mathrm{~mm}$ from the center of the trapping region. The gas expanded between the rods and into the trapping region.

Excitation of the ion of interest was accomplished using dipolar excitation for periods up to $100 \mathrm{~ms}$ and amplitudes up to $80 \mathrm{mV}$. The excitation was at Mathieu $q=0.235$ corresponding to an excitation frequency of $103.5 \mathrm{kHz}$. The ion of interest $(138 \mathrm{~m} / \mathrm{z})$ was produced using a $10 \mathrm{pg} / \mu \mathrm{L}$ solution of caffeine (50/50 methanol/ water plus $0.1 \%$ formic acid) infused at $7 \mu \mathrm{L} / \mathrm{min}$. The $138 \mathrm{~m} / \mathrm{z}$ ion was produced by collision assisted dissociation of the parent caffeine ion $(195 \mathrm{~m} / \mathrm{z})$ injected at a collision energy of $25 \mathrm{eV}$ into the collision cell.

\section{Results and Discussion}

A comparison was made of the MS/MS efficiency of the $m / z 138 \rightarrow 110$ transition of caffeine with and without the pulsed valve activated. As part of this study it was necessary to collect MS/MS data as a function of excitation amplitude and excitation period. It was also necessary to measure and understand the characteristics of the expansion of the gas from the pulsed valve. This was accomplished with the use of a fast ionization gauge (FIG; Jordan TOF Products, Inc., Grass Valley, CA, USA).

\section{Temporal Pressure Profiles}

Temporal pressure profiles of the expanding gas from the pulsed valve were measured on a separate instrument (a modified 4000 QTRAP mass spectrometer) using a FIG. The 4000 QTRAP mass spectrometer was used because its trapping region was more readily accessible to the FIG. The FIG was located $31 \mathrm{~mm}$ from the aperture of the pulsed valve. Figure 2 shows profiles of the expanding gas as a function of the valve open period ranging from 1 to $20 \mathrm{~ms}$. The base pressure was $1.2 \mathrm{e}-5$ Torr for this set of experiments. The profiles in Figure 2a indicate that the pulsed valve opens and the gas expansion develops in less than a millisecond. The profiles also indicate that the valve closes on a submillisecond time scale. The time required for the gas expansion to become fully developed is less than a microsecond implying that the expansion is fully formed shortly after the valve has opened [9].

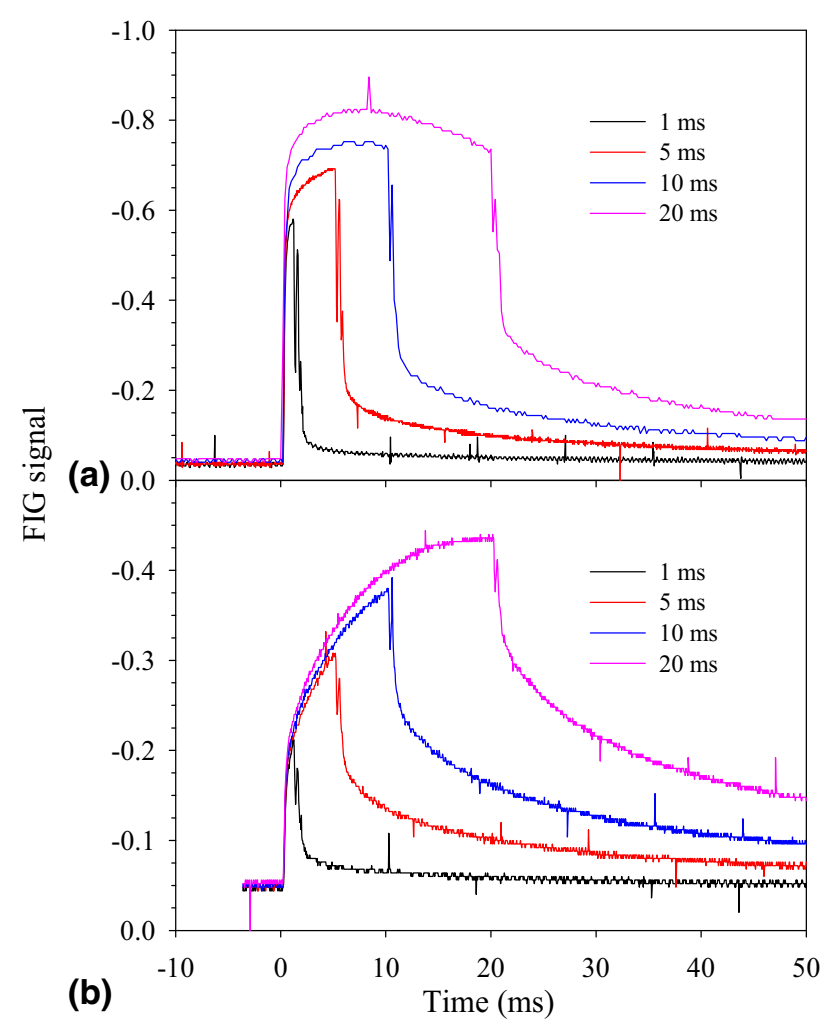

Figure 2. Experimental temporal pressure profiles obtained using a fast ionization gauge (FIG). (a) The FIG is located $31 \mathrm{~mm}$ from the pulsed valve and (b) with the FIG located $45 \mathrm{~mm}$ from the pulsed valve with a pair of quadrupole rods interposed between the pulsed valve and the FIG. 
After the valve has closed the gas requires a period of time before the pressure decreases back to the base pressure. The rate of return to the base pressure depends upon the pump speed and chamber volume in that particular region of the vacuum system. In this case the pump speed and chamber volumes were similar for both instruments so it can be expected that the rates of return to the base pressure will be similar. In the experiments detailed here, $100 \mathrm{~ms}$ was found to be sufficiently long enough to allow the pressure to decrease back to the base pressure. This time period was also sufficiently long enough to allow ions to become thermalized. This was based upon measurements of the peak widths, intensities, and positions in the mass spectrum, which are stable once the ions have become thermalized.

Figure $2 \mathrm{~b}$ shows that when a pair of rods is inserted between the pulsed valve and the FIG the profiles become rounded on the leading edge. The presence of the rods interferes with the expansion causing the pressure to rise less rapidly in the trapping region. The absolute magnitude of the signal was reduced by almost a factor of two when the rods were interposed between the pulsed valve and the FIG. This is because it was necessary to move the FIG to a distance of $45 \mathrm{~mm}$ away from the pulsed valve aperture due to physical limitations imposed by the set-up. A calculation of the pressure decrease, using the equations for a static free jet expansion [10], shows that the pressure will drop by a factor of 2.1 for this change in distance from the origin of the expansion.

Calculation of the pressure within the trapping region indicates that the pressure is about $6.0 \mathrm{e}-4$ Torr. The pressure may in fact be higher due to the confining effects of the quadrupole rods. Normalizing the data of Figure $3 \mathrm{a}$ to the base pressure of $1.2 \mathrm{e}-5$ Torr leads to a maximum pressure of $1.9 \mathrm{e}-4$ Torr with the FIG located $31 \mathrm{~mm}$ from the valve opening. Correcting for the difference in distance, $22 \mathrm{~mm}$ versus $31 \mathrm{~mm}$, gives another factor of 1.99 which brings the pressure up to $3.8 \mathrm{e}-4$ Torr at $22 \mathrm{~mm}$ from the valve opening. The numbers from both the FIG measurements and the calculations suggest that the local pressure within the LIT is between $3.8 \mathrm{e}-4$ and $6.0 \mathrm{e}-4$ Torr.

\section{Resonant Excitation Profiles}

Dipolar excitation was used to excite the ion of interest. Resonant excitation profiles were measured before collection of the MS/MS data to ensure that the ion was on resonance. This was done by monitoring the intensity of the parent ion as the drive rf amplitude (excitation mass) was stepped over a range with the excitation frequency held constant. The excitation amplitude was adjusted in each resonance profile experiment to give $\sim 60 \%$ to $90 \%$ maximum depletion of the ion signal. The location of the resonance remained unaffected with changes in pressure.

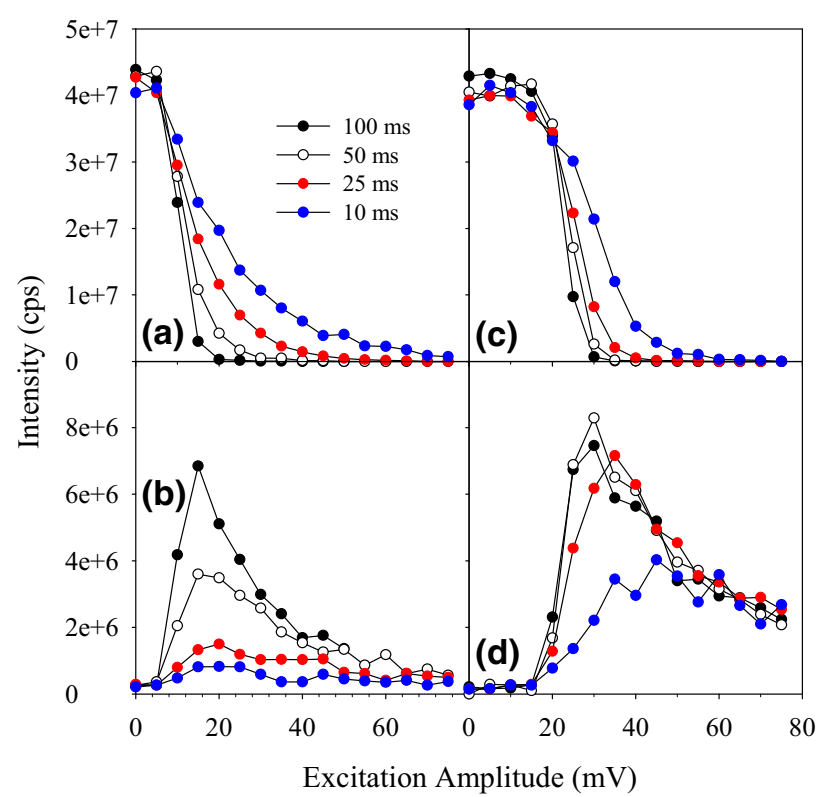

Figure 3. Signal for the precursor ion $138 \mathrm{~m} / \mathrm{z}$ of caffeine at (a) low-pressure and (c) high-pressure and for the $110 \mathrm{~m} / \mathrm{z}$ fragment ion at (b) low-pressure and (d) high-pressure as a function of excitation amplitude and period.

\section{Effect of Excitation Amplitude, Excitation Period, and Pressure}

Figure 3 shows plots of the precursor and fragment ion intensities as a function of excitation amplitude, excitation period and pressure for the $m / z \quad 138 \rightarrow 110$ transition of caffeine excited at $q=0.235$. Figure $3 a$ and $b$ show data with the pulsed valve not activated while Figure $3 c$ and $d$ show data with the valve activated. Excitation periods were varied from 10 to $100 \mathrm{~ms}$. The onset of fragmentation occurred at a higher excitation amplitude (15 to $20 \mathrm{mV}$, Figure 3c) when the pulsed valve was activated than when it was not activated ( 5 to $10 \mathrm{mV}$, Figure 3a). This is a reflection of the increased damping provided by the collision gas. When the pulsed valve was not activated the maximum MS/MS signal dropped by a factor of four as the excitation period was decreased from 100 to $25 \mathrm{~ms}$ and a further factor of two when reduced to $10 \mathrm{~ms}$, Figure $3 \mathrm{~b}$. In contrast, when the pulsed valve was activated the maximum MS/MS signal remained high as the excitation period was reduced to $25 \mathrm{~ms}$, Figure $3 \mathrm{~d}$. It was not until the excitation period was reduced to $10 \mathrm{~ms}$ that a significant decrease occurred. The result is efficient fragmentation in a shorter time period leading to an overall increase in duty cycle.

Figure 4 shows an example MS/MS spectrum of the $138 \mathrm{~m} / \mathrm{z}$ precursor fragmented using an excitation amplitude of $25 \mathrm{mV}$ and excitation period of $25 \mathrm{~ms}$ with the pulsed valve activated. The MSMS efficiency [(sum of the intensity of the products)/(intensity of the precursor before activation)] for this spectrum is $43 \%$. 


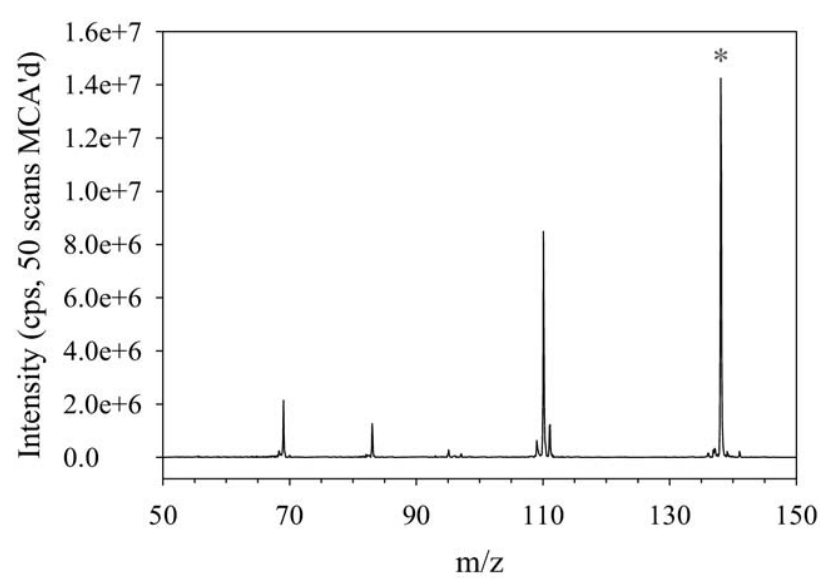

Figure 4. MS/MS spectrum of the $138 \mathrm{~m} / \mathrm{z}$ fragment of caffeine using an excitation amplitude of $25 \mathrm{mV}$ and an excitation period of $25 \mathrm{~ms}$ with the pulsed valve activated. MS/MS efficiency is $43 \%$. The precursor ion is denoted by the asterisk.

\section{Conclusions}

The use of a pulsed valve to increase the local pressure in the low-pressure LIT has allowed excitation periods to be reduced from $100 \mathrm{~ms}$ to $25 \mathrm{~ms}$ without suffering losses in MS/MS efficiency during the excitation process. The reduction in excitation period also results in an increase of the overall duty cycle of the scan.

\section{Acknowledgments}

The authors thank Sasha Loboda for providing the pulsed valve and driver for the initial stages of these studies.

\section{References}

1. Collings, B. A. Fragmentation of Ions in a Low Pressure Linear Ion Trap. J. Am. Soc. Mass Spectrom. 2007, 18, 1459-1466.

2. Collings, B. A. Increased Fragmentation Efficiency of Ions in a Low Pressure Linear Ion Trap with an Added dc Octopole. J. Am. Soc. Mass Spectrom. 2005, 16, 1342-1352.

3. Michaud, A.; Frank, A.; Ding, C.; Zhao, X.; Douglas, D. J. Ion Excitation in a Linear Quadrupole Ion Trap with an Added Octopole Field. J. Am. Soc. Mass Spectrom. 2005, 16, 835-849.

4. Charles, M. J.; McLuckey, S. A.; Glish, G. L. Competition Between Resonance Ejection and Ion Dissociation during Resonant Excitation in a Quadrupole Ion Trap. J. Am. Soc. Mass Spectrom. 1994, 5, 1031-1041.

5. Coopersmith, B. I.; Yost, R. A. Internal Pulsed Valve Sample Introduction on a Quadrupole Ion Trap Mass Spectrometer. J. Am. Soc. Mass Spectrom. 1995, 6, 976-980.

6. Carlin, T. J.; Freiser, B. S. Pulsed valve Addition of Collision and Reagent Gases in Fourier Transform Spectrometry. Anal. Chem. 1983, 55, 571-574.

7. Loboda, A.; Krutchinsky, A.; Loboda, O.; McNabb, J.; Spicer, V.; Ens, W.; Standing, K. Novel. LINAC II Electrode Geometry for Creating an Axial Field in a Multipole Ion Guide. Eur. J. Mass Spectrom. 2000, 6, 531-536.

8. Londry, F. A.; Hager, J. W. Mass Selective Axial Ion Ejection from a Linear Quadrupole Ion Trap. J. Am. Soc. Mass Spectrom. 2003, 14, 1130-1147.

9. Morse, M. D. Supersonic Beam Sources. In Methods of Experimental Physics Vol. II. Atoms and Molecules. Dunning, F. B.; Hulet, R. Eds. Academic Press: Orlando 1996; p. 21-47.

10. Ashkenas, H.; Sherman, F. S. In Rarefied Gas Dynamics. 4th Symposium Vol. II. de Leeuw, J. H., Ed.; Academic Press: New York, 1966; p. 84-105. 